\title{
CARACTERIZAÇÃO DA OCORRÊNCIA DE PADRÕES DE SONAR DE VARREDURA LATERAL E SÍSMICA DE ALTA FREQÜÊNCIA $(3,5$ e 7,0 kHz) NA PORÇÃO SUL DA BAÍA DE GUANABARA - RJ
}

\author{
V. S. Quaresma1, G. T. M. Dias ${ }^{2}$ \& J. A. Baptista Neto ${ }^{2}$ \\ Received October 12, 1999 / Accepted April 21, 2001
}

\begin{abstract}
A correlação de dados geofísicos (sonar de varredura lateral e perfilador de subfundo - 3,5 e 7,0 kHz) permitiu a caracterização da distribuição de sedimentos de fundo na porção sul da Baía de Guanabara. Foram reconhecidos quatro tipos de ecocaráteres: o tipo 1, onde não ocorre penetração do sinal acústico e está relacionado a um fundo arenoso e a um padrão de reflexão do sonar homogêneo cinza claro com sand wave e mega-ripples; o tipo $2 \mathrm{~A}$, onde é observada a penetração do sinal com refletores do embasamento acústico e um fundo predominantemente lamoso com alto teor de areia fina a muito fina e padrão homogêneo cinza claro de sonar; o tipo 2B, com penetração do sinal com refletores sendo observados junto a um fundo predominantemente arenoso, com alto teor de lama e padrões de sonar homogêneos cinza escuros; e o último ecocaráter observado, o tipo 3 , com penetração do sinal com uma série de refletores múltiplos, observando-se um fundo lamoso com alto teor de areia fina a muito fina e reflexão homogênea cinza escuro nos sonogramas.
\end{abstract}

Palavras-chave: Baía de Guanabara; Sísmica de alta freqüência; Sonar de varredura lateral; Sedimentos de fundo.

CHARACTERIZATION OF SIDE-SCAN SONAR AND HIGH RESOLUTION SEISMIC (3.5 - 7.0 kHZ) REFLECTION PATTERNS ALONG THE SOUTHERN MARGIN OF THE GUANABARA BAY - RJ - The correlation of geophysical data (side-scan sonar and subbottom profiler - 3.5 and $7.0 \mathrm{kHz}$ ) has enabled the characterization of the sediment distribution along the southern portion of the Guanabara Bay (Rio de Janeiro - Brazil). Four distinct echocharacter types were recognized: type 1 shows no penetration of the acoustic signal, it is associated with a sandy bottom and with a light gray homogeneous sonograph pattern with sand waves and mega ripples; type $2 A$ presents internal reflectors and the acoustic basement, it is related to a muddy bottom with high content (\%) of fine sand and to light gray homogeneous sonograms; type $2 B$ is marked by signal penetration and is related to a sandy bottom with high content (\%) of mud and to dark grey homogeneous sonograms; and, finally, type 3 with signal penetration and multiples, it is associated with a muddy bottom with high content (\%) of fine sand, and is related to dark gray homogeneous sonograph patterns.

Key words: Guanabara Bay; High-frequency seismic; Side-scan sonar; Bottom sediments.

\footnotetext{
${ }^{1}$ School of Ocean and Earth Sciences -Univ. of Southampton Southampton Oceanography Centre, Empress Dock, Southampton, SO14 3ZH, UK

${ }^{2}$ Laboratório de Geologia Marinha -Dept de Geologia /LAGEMAR-UFF

${ }^{2} \mathrm{Av}$. Litorânea,s/no $4^{\circ}$ andar - Gragoatá - Niterói - RJ CEP:24210-340
} 


\section{INTRODUÇÃO}

A Baía de Guanabara é uma das mais proeminentes baías do litoral brasileiro e está localizada no estado do Rio de Janeiro, na região metropolitana da cidade do Rio de Janeiro, entre as latitudes de $22^{\circ} 40^{\prime}$ e $23^{\circ} 00^{\prime}$ Sul e longitude $43^{\circ} 00^{\prime}$ e $43^{\circ} 20^{\prime}$ Oeste (Fig.1). A superfície da baía mede aproximadamente $380 \mathrm{~km}^{2}$, com um volume médio de água em torno de $1,87 \times 10^{9} \mathrm{~m}^{3}$. O seu perímetro é de $131 \mathrm{~km}$, com extensão máxima de $28 \mathrm{~km}$ de leste a oeste e cerca de $30 \mathrm{~km}$ de norte a sul (Kjerfve et al., 1996), sofrendo um estreitamento em sua barra (entre a Fortaleza de Santa Cruz e o Forte de São João), onde nessa área a largura da baía se restringe a 1,6 km (Fig.1).

O sistema da Baía de Guanabara e bacia de drenagem associada está inserido no rifte da Guanabara (Ferrari, 1990) que faz parte do conjunto de depressões Terciárias da costa sudeste brasileira, e tem sua configuração atual ligada às variações do nível do mar, no Holoceno, que condicionaram o afogamento de um antigo vale fluvial Pleistocênico (Amador, 1992). Desta forma, segundo classificação proposta por Perillo (1995), a baía pode ser classificada como um estuário de planície costeira (Quaresma, 1997).

A Baía de Guanabara está localizada numa das regiões mais industrializadas do Brasil, e desta forma tornou-se um dos ambientes estuarinos mais degradados do país. Portanto o entendimento da sua hidrodinâmica e das características do fundo são primordiais para estudos que venham a ser desenvolvidos na área.

Este trabalho tem como objetivo caracterizar a distribuição de sedimentos de fundo através da correlação de dados geofísicos de sonar de varredura lateral e perfilador de subfundo $(3,5$ e $7,0 \mathrm{kHz}) \mathrm{com}$ amostras superficiais de sedimentos, bem como na caracterização dos tipos de ecocaráters e reflexão de sonar encontrados neste ambiente, ou em ambientes semelhantes.

\section{MATERIAL E MÉTODOS}

Para a confecção dos mapas de padrões de reflexão do sonar, foram utilizados registros obtidos durante levantamentos realizados pelo Curso de Especialização em Geologia e Geofísica Marinha do LAGEMAR-UFF, entre os anos de 1988 e 1995. Nestes levantamentos foram realizados perfilagens sísmicas $(36 \mathrm{~km})$ com o sistema Raytheon modelo RTT 1000A e sonografias

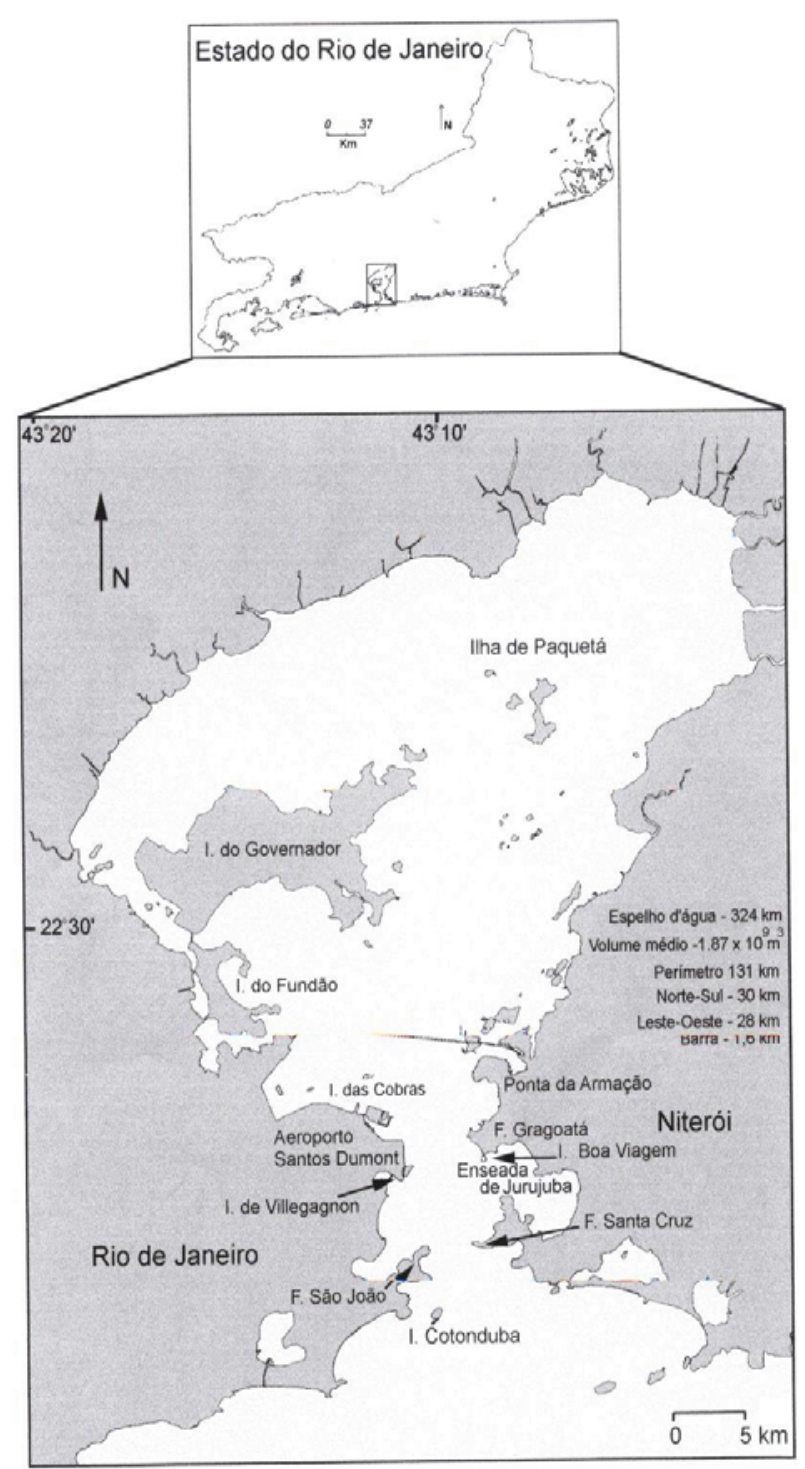

Fig.1: Localização da área de estudo.

Fig.1: Study area

do fundo com o sonar de varredura lateral EGG-260/ 272T. Foram ainda interpretados os sonogramas obtidos pela Diretoria de Hidrografia e Navegação (DHN), num total de $102 \mathrm{~km}$ (Fig.2). O equipamento utilizado foi o Sonar e posicionamento por Radar DECCA.

Os registros de sísmica de alta freqüência, 3,5; 7,5 e $12 \mathrm{kHz}$ têm sido largamente utilizados como ferramentas em geologia marinha nas investigações dos tipos de sedimentos do fundo do mar, geomorfologia submarina e processos sedimentares (Hollister \& Heezen, 1972; Damuth, 1975; Damuth \& Hayes, 1977 apud Flood, 1980). Estes equipamentos operam em freqüências elevadas 3,5 à $12 \mathrm{kHz}$, o que permite uma resolução submétrica dos refletores em subsuperficie em detrimento de uma maior penetração, que em condições ideais, em sedimentos lamosos pode atingir 

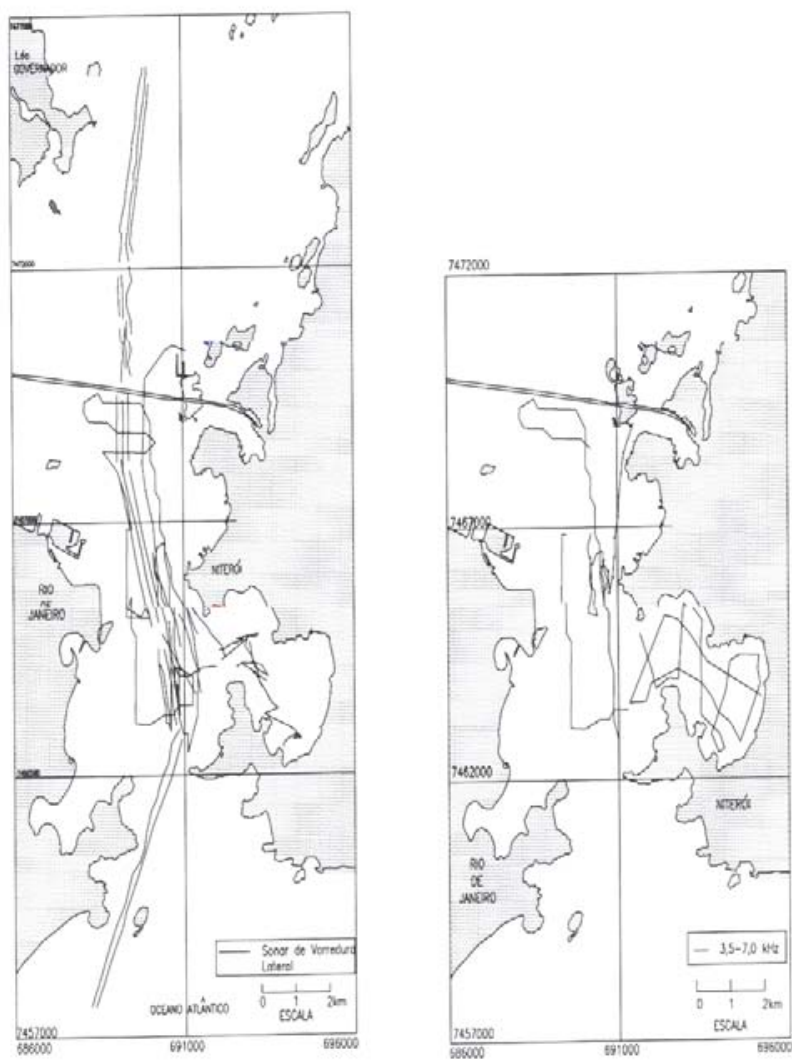

Fig.2: Localização do sonar de varredura lateral a esquerda e das linhas de 3,5 e 7,0 kHz a direita.

Fig.2: Tracks of side-scan sonar (left) and subbottom profiler (right).

mais de 20 metros.

O padrão do eco é resultado da interação entre o fundo oceânico e o pulso da energia usada como fonte. O retorno do eco é afetado pelo tipo de sedimento e suas camadas (coeficiente de reflexão, espessura das camadas, interferência entre as camadas, reflexões internas múltiplas, etc.) e pelas suas estruturas e topografias (difrações, extensão das camadas, variação lateral no espaçamento das camadas, etc.) (Flood, 1980).

O sistema de sonar de varredura lateral se baseia na transmissão de um sinal acústico por um transdutor e sua reflexão pelo fundo marinho, retornando ao transdutor, onde é transformado em pulso elétrico e registrado em um papel térmico ou eletro-sensível (Ayres Neto \& Aguiar, 1993). Os vários tons de cinza que se observam nos registros resultam da intensidade da reflexão do sinal. Essa reflexão se dá em função da textura e morfologia do fundo, da granulometria do sedimento e de características particulares do sedimento como por exemplo o grau de compactação.

Aproximadamente 100 amostras de fundo foram coletadas em diversos levantamentos realizados, cobrindo toda a área estudada (Fig.3). Os sedimentos foram classificados seguindo metodologia proposta por Larsonneur (1977) e modificada por Dias (1996).

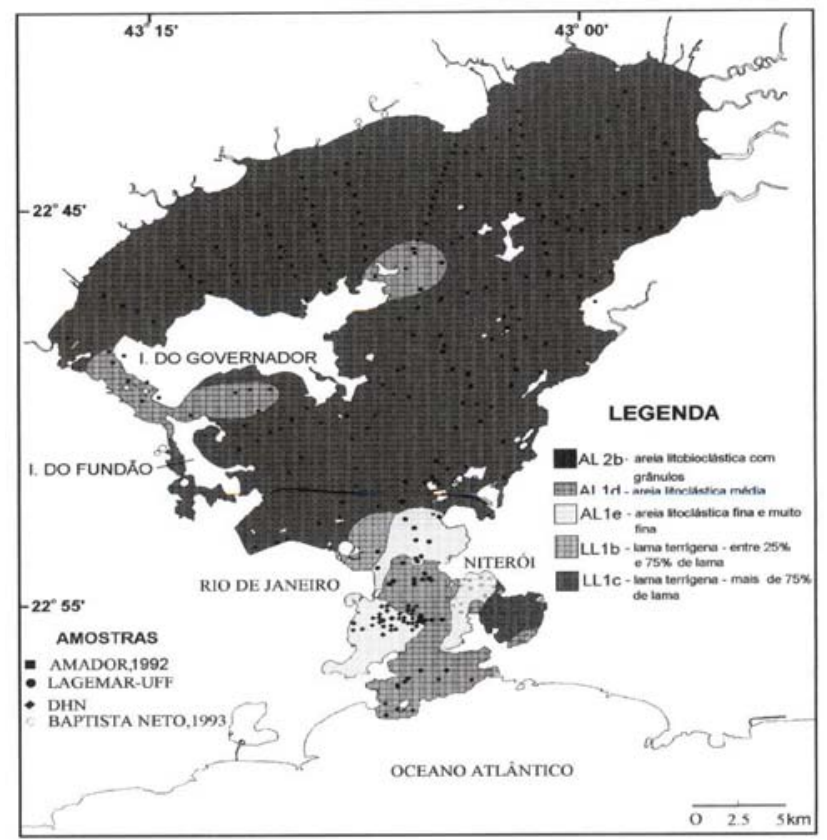

Fig.3: Distribuição dos sedimentos de fundo da Baía de Guanabara (UFF - Universidade Federal Fluminense; DHN Diretoria de Hidrografia e Navegação).

Fig.3: Bottom sediment distribution in the Guanabara Bay (UFF - Universidade Federal Fluminense; DHN - Diretoria de Hidrografia e Navegação)..

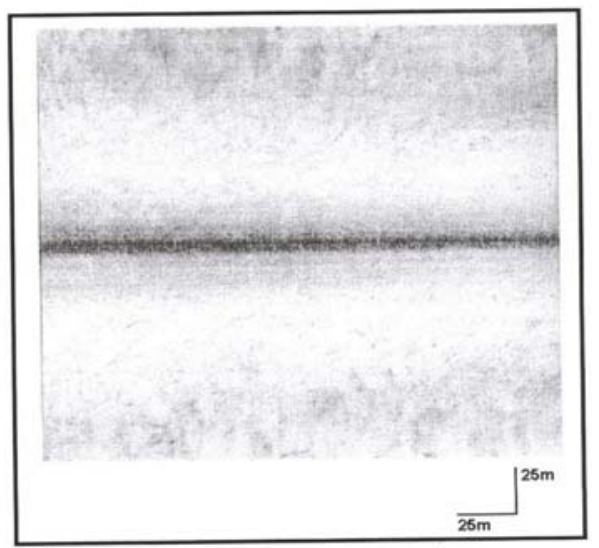

Fig.4: Padrão de reflexão do sonar de varredura lateral homogêneo cinza claro.

Fig.4: Homogeneous light grey reflection pattern of the sidescan sonar. 


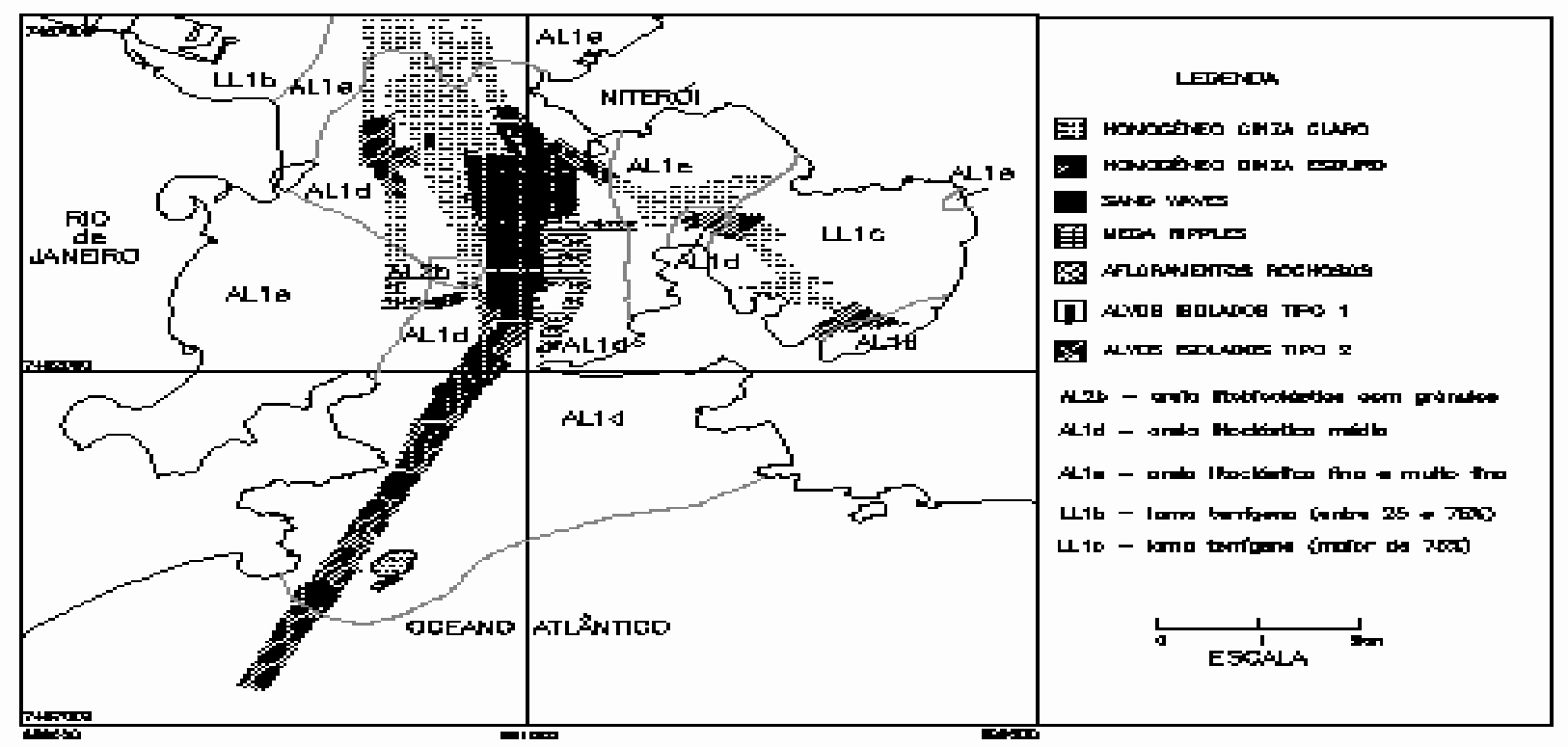

Fig.5: Padrões de reflexão de sonar de varredura lateral com distribuição de sedimentos.

Fig.5: Reflection pattern of side-scan sonar with sediments distribution.

\section{RESULTADOS}

\section{Sonar de Varredura Lateral}

Foram observados padrões de reflexão de sonar de varredura lateral dos tipos: homogêneo cinza claro, homogêneo cinza escuro, sand waves, mega ripples, afloramentos rochosos, alvos isolados.

O padrão homogêneo cinza claro (Fig. 4) é o de maior distribuição dentro da baía, sendo encontrado, principalmente, dentro da enseada de Jurujuba e a norte do alinhamento Forte São João - Fortaleza Santa Cruz (Fig. 5). Os registros são homogêneos e de tonalidade clara. O tipo de fundo relacionado a este padrão é de areia média (AL1d); areia fina (AL1e), em alguns pontos com teor de lama entre $5 \%$ e $15 \%$, e lamas (LL1b e LL1c).

Em outros locais este tipo de reflexão aparece relacionado a lamas e areias finas a muito finas, semelhante ao observado na bacia de Campos (Ayres Neto \& Aguiar,1993) e no Parcel do Carpinteiro no Rio Grande do Sul (Calliari et al.,1994). Neste trabalho, este tipo de reflexão também aparece associado a um fundo de areia média (AL1d), sendo que nessas áreas as areias apresentam teor de areia fina a muito fina entre 14 e $40 \%$.

O registro que apresenta o padrão homogêneo cinza escuro mostra, textura homogênea e tonalidade de um cinza médio a escuro (Fig. 6). Esse padrão se encontra associado a um fundo de areia média (AL1d) sendo encontrado na parte externa da Baía de Guanabara, dentro da enseada de Jurujuba, nas proximidades da Ilha da Boa Viagem e próximo ao aeroporto Santos Dumont. Ainda é observado em área de fundo lamoso e de areia fina a muito fina (Figs. $5 \mathrm{e}$ 7). De acordo com Dias, G.T.M. (comunicação pessoal) ao largo de Cabo Frio areias muito finas lamosas compactadas apresentam padrão de reflexão semelhante.

Os padrões de sand waves e mega ripples (Fig. 8) são comuns na entrada da baía, sendo mais freqüentes no flanco leste do canal central, sendo encontrados ainda na área externa da baía (Fig. 5). Observa-se que

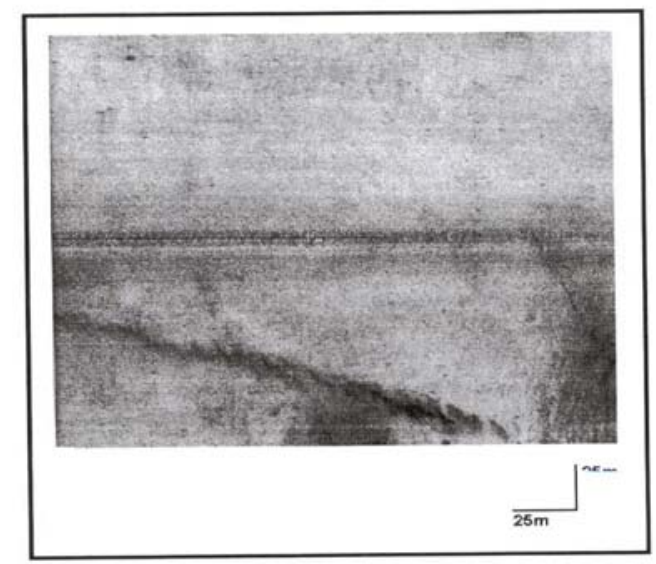

Fig.6: Padrão de reflexão do sonar de varredura lateral homogêneo cinza escuro

Fig.6: Homogeneous dark grey reflection pattern of the sidescan sonar. 


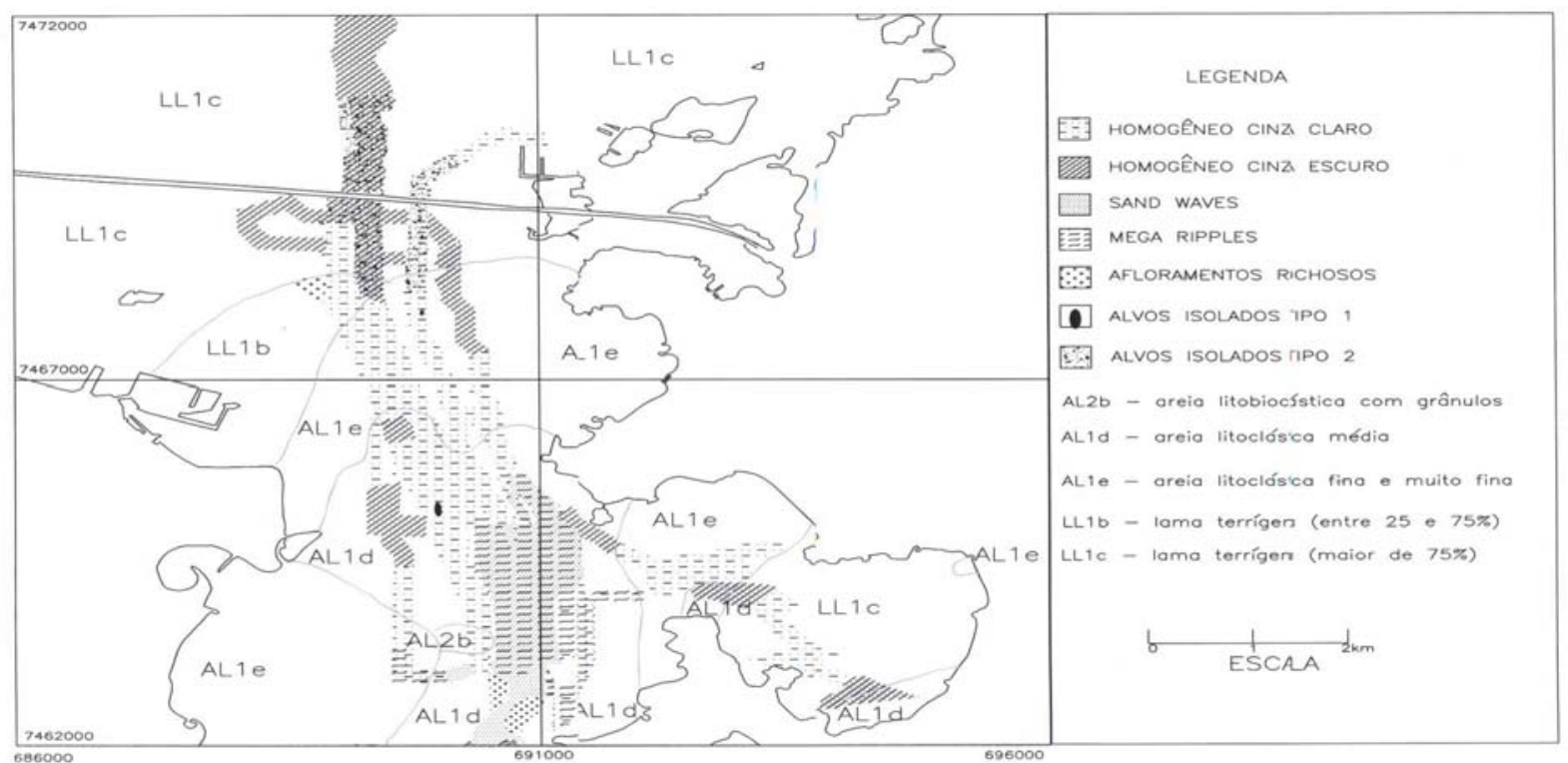

Fig.7: Padrões de reflexão de sonar de varredura lateral com distribuição de sedimentos. Fig.7: Reflection pattern of side scan-sonar with sediments distribution.

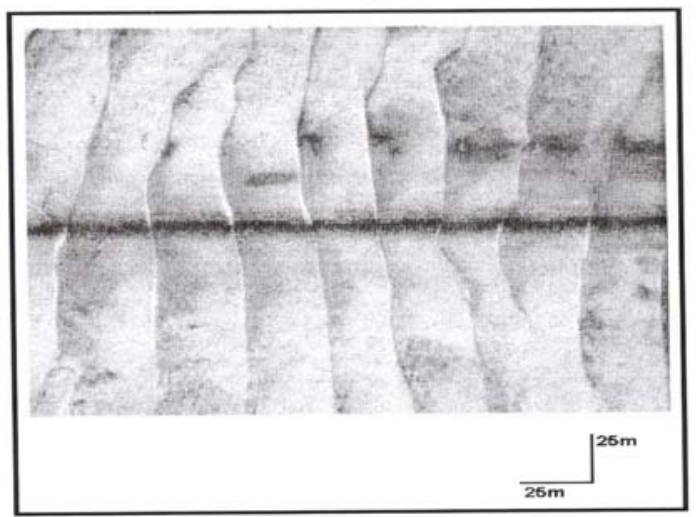

Fig.8: Padrão de reflexão do sonar de varredura lateral mostrando sand waves.

Fig.8: Reflection pattern of side-scan sonar showing sand waves.

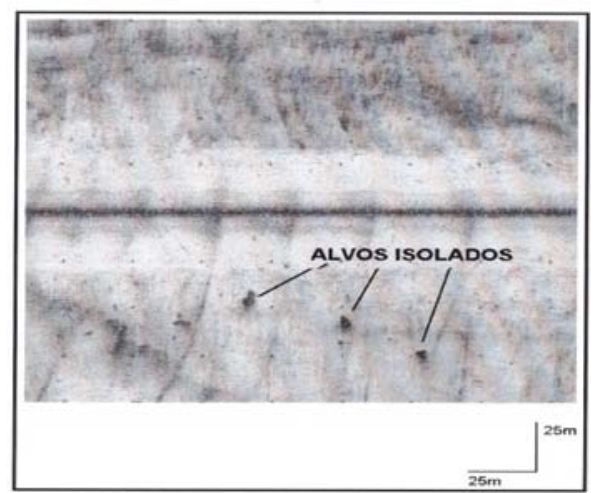

Fig.10: Padrão de reflexão do sonar de varredura lateral de alvos isolados 1 .

Fig.10: Reflection pattern of side-scan sonar of isolated targets1.

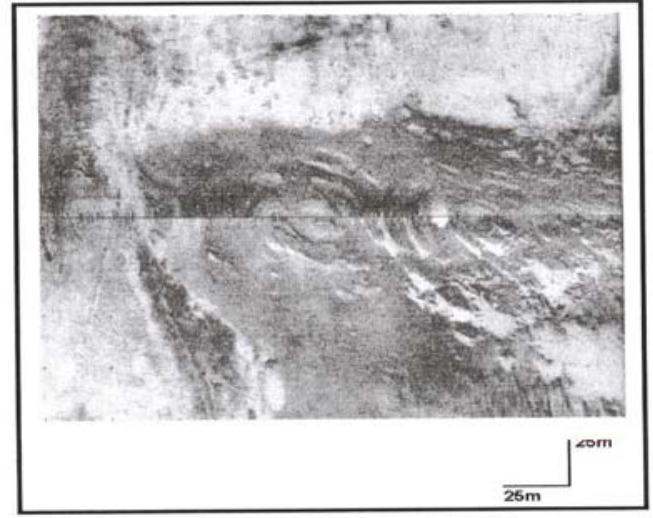

Fig.9: Padrão de reflexão do sonar de varredura lateral mostrando afloramentos rochosos.

Fig.9: Reflection pattern of side-scan sonar showing rock outcrops.

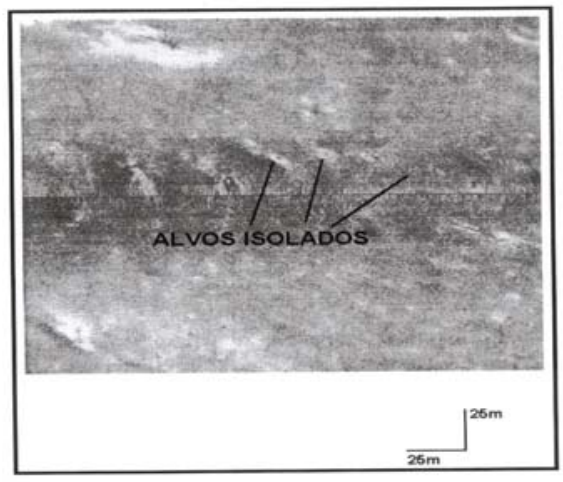

Fig.11: Padrão de reflexão do sonar de varredura lateral de alvos isolados2.

Fig.11: Reflection pattern of side-scan sonar of isolated targets 2 . 
as mega-ripples ocorrem sobre as sand waves, e que a orientação das cristas dessas formas de fundo são perpendiculares à direção da corrente de maré. Esse tipo de reflexão ocorre em fundos arenosos de areias médias (AL1d).

O registro de afloramentos rochosos tem por característica uma alternância de reflexões claras e escuras e textura irregular (Fig. 9). São observados principalmente na entrada da Baía de Guanabara entre o Forte São João e a Fortaleza Santa Cruz, na área externa da baía a leste do Pão de Açúcar, a oeste da Ilha de Cotunduba e a leste da Ilha do Governador (Fig. $5)$.

O padrão de alvos isolados tipo 1 apresenta pequenos pontos de manchas escuras que ocorrem aleatoriamente (Fig. 10), não têm formas preferenciais e ficam entre 10 e $100 \mathrm{~m}$ de comprimento, podendo estar relacionados a estruturas artificiais, cardumes, objetos metálicos, etc., não se relacionando a um fundo preferencial.

Alvos pontuais de pequenas dimensões comprimento (até $1 \mathrm{~m}$ ), denominados alvos isolados tipo 2 (Fig. 11), são encontrados somente a cerca de $1200 \mathrm{~m}$ ao sul e a norte da ponte Rio-Niterói, provavelmente relacionados a detritos resultantes das obras de construção da ponte. (Fig. 7)

\section{Sísmica Rasa (3,5 e 7,0 kHz)}

Com relação aos registros de sísmica rasa $3,5 \mathrm{kHz}$, foram reconhecidos quatro padrões distintos ao longo da área levantada, sendo utilizada a classificação proposta por Baptista Neto (1993) e Baptista Neto et al. (1996), em trabalho realizado na Enseada de Jurujuba. $\mathrm{O}$ autor (op cit) classifica três tipos distintos de ecocaráter, em tipo 1, tipo 2 e tipo 3 .

O ecocaráter do tipo 1 é caracterizado por não ocorrer penetração no subfundo, estando relacionado a um sedimento arenoso (Fig. 12). No tipo 2 tem-se a penetração do sinal e consegue-se observar refletores de subfundo e o embasamento acústico, estando relacionado a um sedimento lamoso (Fig. 13). O ecocaráter do tipo 3 também ocorre com uma penetração do sinal apresentando uma série de refletores múltiplos (Fig. 14) estando relacionado a uma área de transição areia-lama indicando ainda a presença de gás no sedimento.

Neste trabalho foram encontrados os três tipos de ecocaráteres estabelecidos por Baptista Neto (1993) e um quarto tipo, que seria uma variação do tipo 2 deste autor. Dessa forma o tipo 2 passa a ser chamado de $2 \mathrm{~A}$ e a variação encontrada como $2 \mathrm{~B}$.

$\mathrm{O}$ ecocaráter do tipo $2 \mathrm{~B}$ mostra uma penetração do sinal no subfundo, tendo como característica a ocorrência de refletores, e não se observa o embasamento acústico (Fig. 15).

Como pode ser observado na Fig. 16, o ecocaráter do tipo 1 é encontrado da entrada da Baía até cerca de $350 \mathrm{~m}$ a norte da Ilha da Boa Viagem, próximo ao aeroporto Santos Dumont, e na parte ocidental da Enseada de Jurujuba, estando relacionado a um fundo de areia média (AL1d). Observa-se ainda, na margem leste do canal central, que o campo de sand waves não continua a partir das proximidades da Ilha da Boa Viagem. Nessa área nota-se uma passagem gradual das areias médias para as areias finas a muito finas (Fig. 17), além de uma suavização do fundo que passa a ter uma característica plana. Esta suavização também é observada na margem oeste do mesmo canal.

$\mathrm{O}$ ecocaráter do tipo $2 \mathrm{~A}$ é observado a partir de $350 \mathrm{~m}$ a NW da Ilha da Boa Viagem se estendendo até a ponta da Armação (Fig. 6). Essa área está relacionada a um fundo plano de areias médias (AL1d) e de areias finas a muito finas (AL1e). Na Enseada de Jurujuba, segundo Baptista Neto (1993), esse tipo de ecocaráter estaria localizado na parte oriental da enseada, estando relacionado a um fundo lamoso. Neste caso foram feitas algumas considerações sobre as amostras de sedimento de fundo coletadas na área fora da enseada, onde as areias médias apresentam um teor de areia fina a muito fina entre 10 e $40 \%$, notando-se ainda que o embasamento acústico está bem mais raso do que na enseada. Pode ser, em função destes aspectos, a ocorrência deste tipo de ecocaráter em área com sedimento de fundo diferente do encontrado na Enseada de Jurujuba.

O fundo relacionado a esse tipo de ecocaráter se mostra plano, apresentando um canal com variação de cerca de $3 \mathrm{~m}$ na profundidade (Fig. 18), ao largo do Forte de Gragoatá. Esse canal também pode ser observado no mapa batimétrico (Fig. 19) e, de acordo com o mapa geológico do DRM, dá seqüência a uma falha mapeada. Alguns perfis foram feitos na área, apesar de não apresentarem uma boa resolução e estarem em escalas diferentes, pode-se observar a continuidade dessa falha em direção ao canal central, onde se nota que o embasamento está bem próximo à superfície, tendo morfologia típica de "Pão de Açúcar", como em outras áreas na entrada da Baía onde o embasamento cristalino aflora (Fig. 19).

$\mathrm{O}$ ecocaráter do tipo $2 \mathrm{~B}$ aparece logo após a 


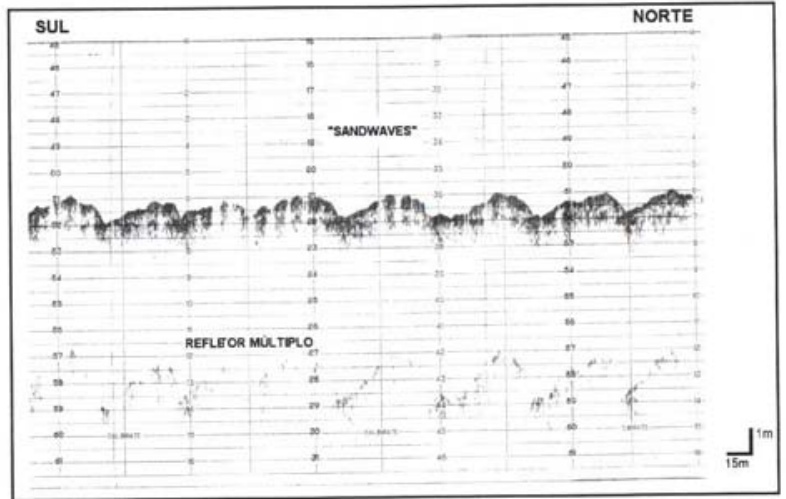

Fig.12: Ecocaráter do tipo 1. Fig.12: Echocharater type 1.

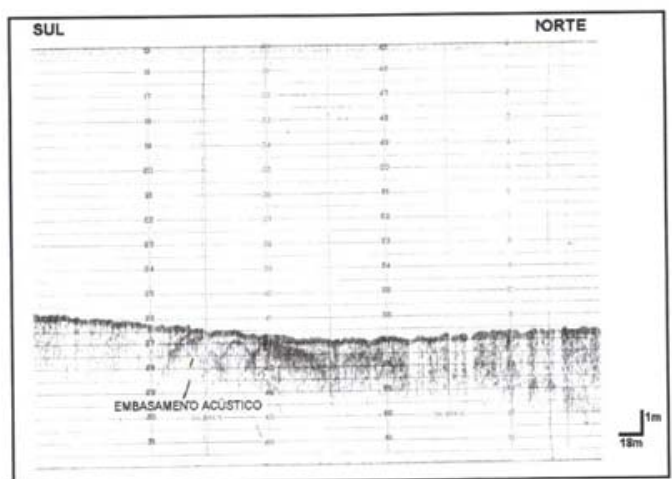

Fig.13: Ecocaráter do tipo 2. Fig.13: Echocharater type 2.

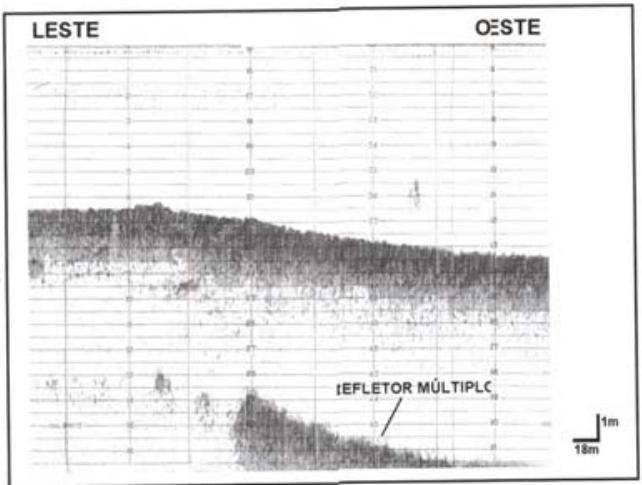

Fig.14: Ecocaráter do tipo 3. Fig.14: Echocharater type 3.

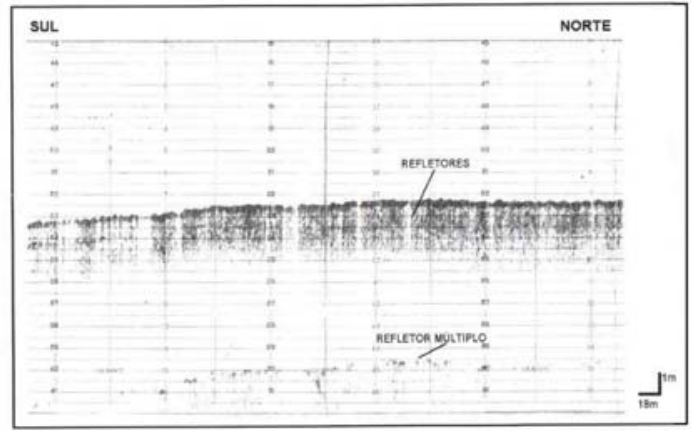

Fig.15: Ecocaráter do tipo 2B.

Fig.15: Echocharater type 2B. 


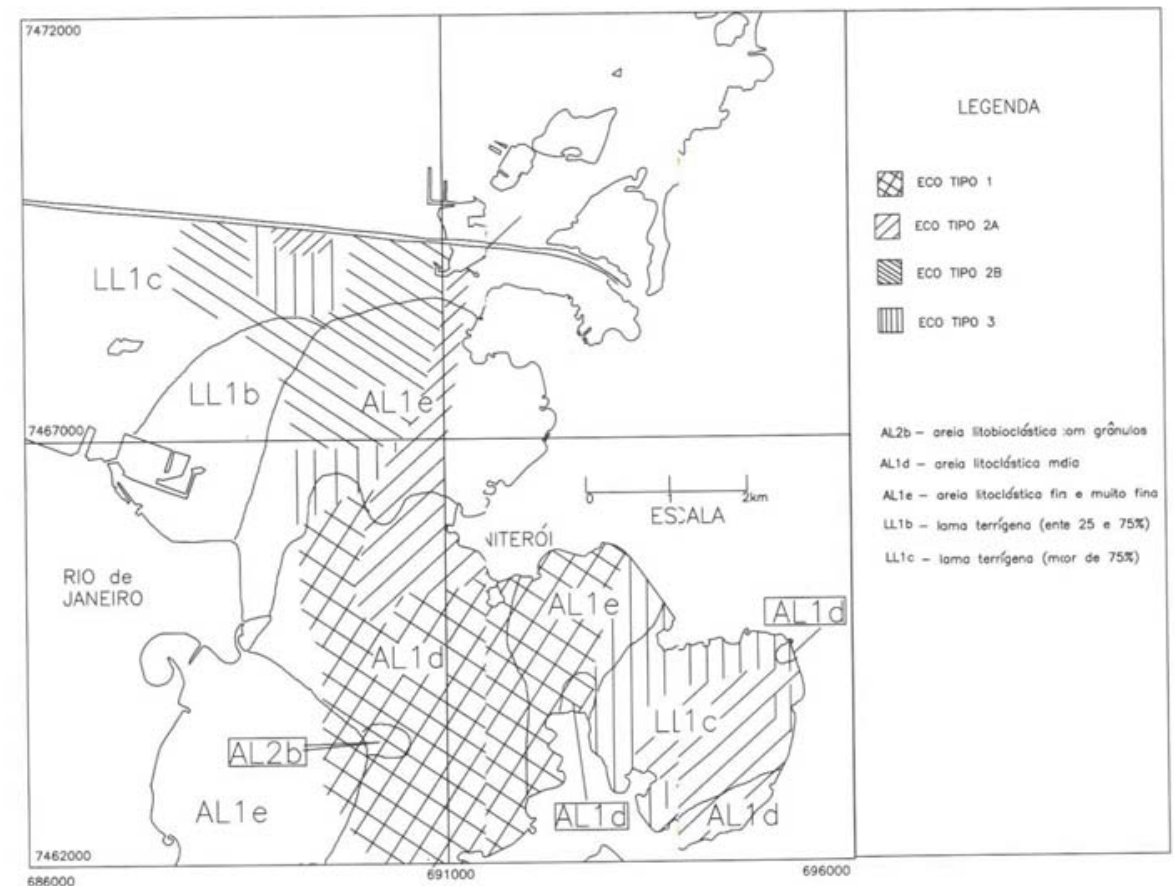

Fig.16: Mapa de ecocaráter com distribuiçào de sedimentos.

Fig.16: Echocharater map with sediment distribution

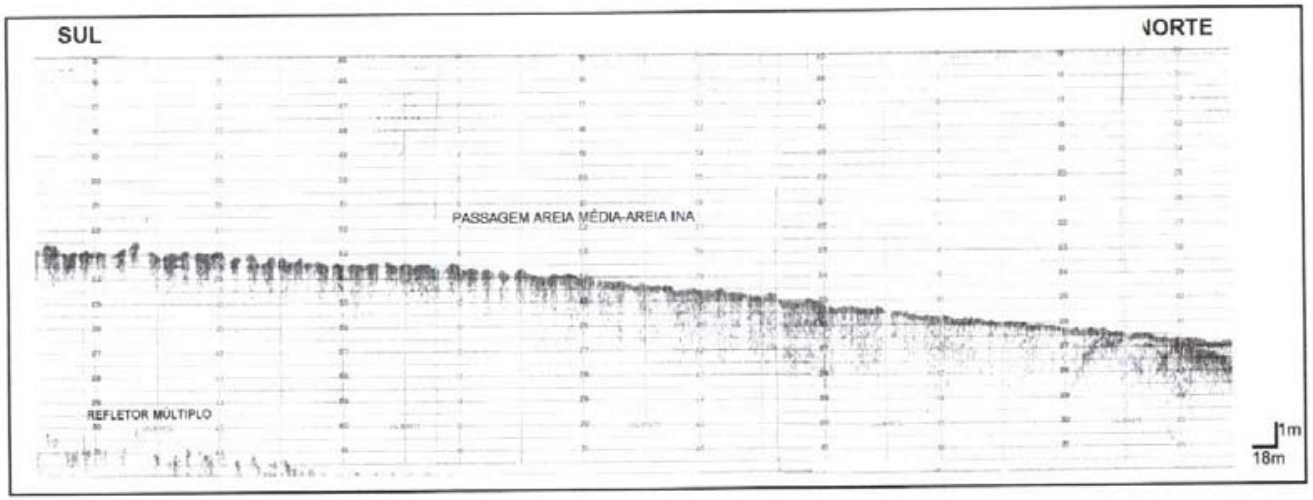

Fig.17: Perfil de 3,5 kHz mostrando a passagem de areia média para areia fina e muito fina.

Fig.17: Subbottom profile showing the limit of medium sand to fine and very fine sand.

ocorrência do ecocaráter $2 \mathrm{~A}$, sendo observado a partir da Ilha das Cobras até a ponte Rio-Niterói (Fig. 16). Está relacionado a um fundo plano de areia fina a muito fina (AL1e) e de lama (LL1b e LL1e), onde a profundidade se mantém com uma certa regularidade. Observa-se a ocorrência de refletores planos e irregulares .

O ecocaráter do tipo 3 é observado entre a Ilha das Cobras e o aeroporto Santos Dumont, numa pequena área próxima ao vão central da ponte e na parte central da Enseada de Jurujuba (Fig. 16). Esse tipo de ecocaráter está relacionado a um fundo lamoso, com teor de areia fina de cerca de $20 \%$. Próximo ao vão central o registro se mostra com uma forte reflexão, mascarando os refletores de subfundo (Fig. 5), estas anomalias acústicas são características da presença de gás no sedimento que é produzido pelo processo de decomposição da matéria orgânica desencadeado por bactérias anaeróbicas. Vários autores já descreveram essas anomalias em outras áreas tanto em regiões marinhas profundas (Figueiredo Jr. et al.,1993; Manley \& Flood, 1989 e Tucholke et al., 1977) como em áreas protegidas como os estuários (Baptista Neto, 1993). 


\section{DISCUSSÃO}

Quando se faz uma comparação entre a distribuição do padrão de reflexão do sonar de varredura lateral, os registros de $3,5 \mathrm{kHz}$ e os sedimentos de fundo, nota-se que há uma boa correlação dessas informações.

A Tab. 1 mostra a relação entre os diversos tipos de ecocaráter com o tipo de sedimento. $\mathrm{O}$ ecocaráter do tipo1 está relacionado a um fundo arenoso onde cerca de $60 \%$ das amostras analisadas são de areias médias com um alto teor de areias finas e $40 \%$ são areias finas e muito finas. Este padrão de ecocaráter é encontrado em área onde a reflexão do sonar se mostra como homogêneo cinza claro ou apresenta o padrão relacionado às sand waves e mega-ripples (Figs. $5 \mathrm{e}$
16). Nessa área da baía de Guanabara ocorreram as maiores velocidades das correntes de maré, os campos de dunas subaquosas migram para o interior da baia condicionadas por fortes correntes de preamar e ondas de SE (Dias \& Quaresma, 1996)

O ecocaráter do tipo $2 \mathrm{~A}$ também está relacionado, principalmente, aos sonogramas com reflexão do tipo homogêneo cinza claro, onde se tem sedimentos lamosos (LL1c e LL1e), representado por cerca de $65 \%$ das amostras, e sedimentos arenosos dos tipos areias médias, em torno de $20 \%$ das amostras, que apresentam alto teor de areia fina (entre 15 e $40 \%$ ) e ainda areias finas e muito finas (AL1e) em torno de 15\% (Tab. $1 \mathrm{e}$ Figs. 5 e 16). Provavelmente, o teor de sedimentos de granulometria fina nas amostras explica a penetração do sinal em áreas com fundo arenoso. Esse ecocaráter

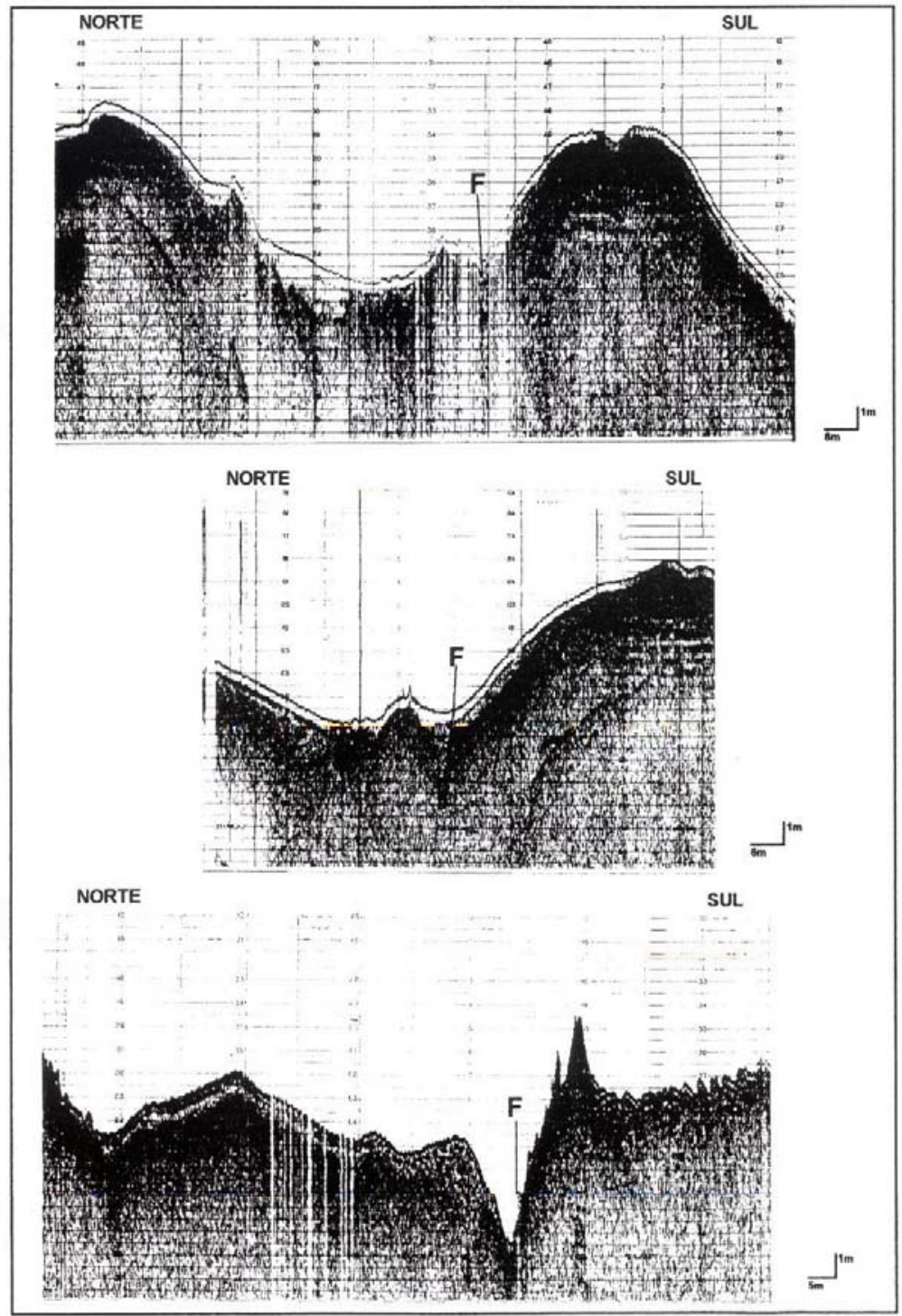

Fig.18: Perfis mostrando uma feição possivelmente associada ao prolongamento de uma falha (F) mapeada no continente em DRM, 1981.

Fig.18: Profile showing a feature possibly associated with a fault (F) mapped in the continent (DRM, 1981). 
e a distribuição de sedimentos mostra nitidamente uma diminuição da energia hidrodinâmica, quando se acompanha a passagem do ecocaráter tipo 1 para o tipo 2A (Fig. 9).

O ecocaráter do tipo $2 \mathrm{~B}$ está relacionado, na sua maior parte, a uma reflexão homogênea cinza escuro tendo como fundo, areias finas a muito finas e lamas (Figs. 5 e 16). Esse ecocaráter tem maior ocorrência em direção à ponte Rio-Niterói onde a tendência da granulometria do sedimento é se tornar cada vez mais fina. Não se consegue observar o embasamento acústico nesse tipo de ecocaráter, provavelmente devido ao fato de se encontrar a maiores profundidades, observando-se apenas refletores que provavelmente mostram variações litológicas ou diferentes graus de compactação do sedimento.

O ecocaráter do tipo 3 é observado, principalmente, sobre fundo lamoso e em áreas de transição areia-lama e com presença de gás, estando relacionado, também, a um padrão de reflexão homogêneo cinza escuro nos sonogramas (Figs. 5 e 16). Como pode ser observado na Tab. 1, as lamas relacionadas a esse tipo de ecocaráter apresentam altos teores de areia fina a muito fina, algumas vezes chegando a $30 \%$, o que, dessa forma, caracteriza bem a passagem de um fundo arenoso para um fundo lamoso.

Como descrito anteriormente, as anomalias acústicas observadas na área próxima à ponte RioNiterói são típicas de áreas que apresentam gás no

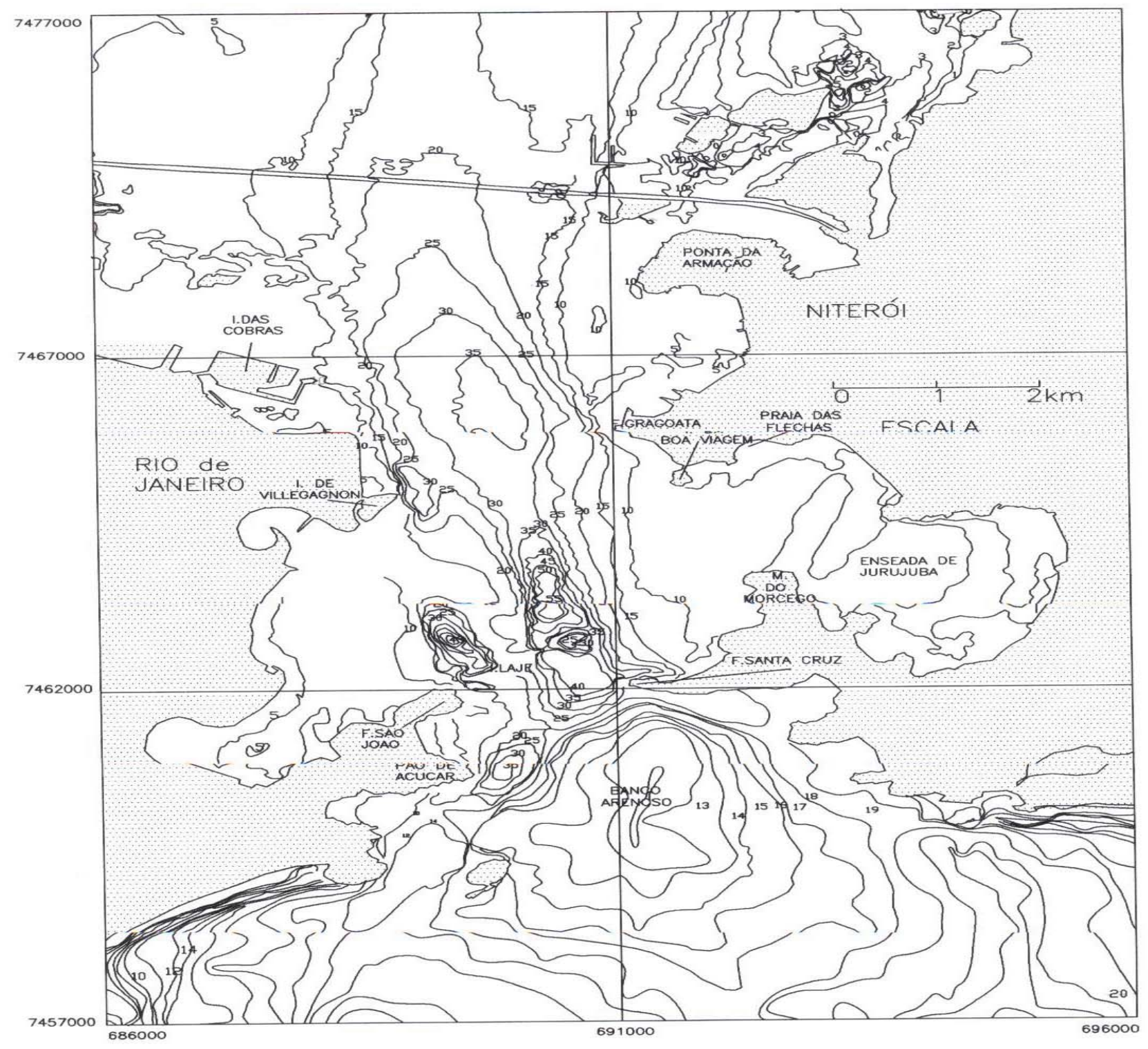

Fig.19: Mapa de batimetria da Baía de Guanabara.

Fig.19: Bathymetric map of the Guanabara Bay. 


\begin{tabular}{|c|c|c|c|c|}
\hline TIPO DE ECO & $\begin{array}{c}\text { CLASSIFICACCAOO E } \\
\text { PORCENTAGEM TOTAL } \\
\text { DE AMOSTRAS } \\
\text { CORRESPONDENTES }\end{array}$ & $\begin{array}{l}\text { AREIA } \\
\text { FINA(\%) }\end{array}$ & LAMA(\%) & FUNDO ASSOCIADO \\
\hline \multirow{3}{*}{$\begin{array}{l}\text { (s/ pene tração do } \\
\text { sinal acústico) }\end{array}$} & AL1d $(61 \%)$ & $1-40$ & $0-8$ & Fundo arenoso \\
\hline & AL1e $(39 \%)$ & $55-75$ & $0-2,5$ & \\
\hline & LL1c $(65 \%)$ & $1-10$ & $85-99$ & \\
\hline $2 \mathrm{~A}$ & AL1d $(20 \%)$ & $15-40$ & $0-9$ & $\begin{array}{l}\text { Fundo lamoso com } \\
\text { alto teor de areia fina } \\
\text { a muito fina }\end{array}$ \\
\hline \multirow{3}{*}{$\begin{array}{l}\text { (c/ pene tração do } \\
\text { sinal observando-se } \\
\text { refletores e o } \\
\text { embasamento } \\
\text { acústico) }\end{array}$} & AL1e $(15 \%)$ & $45-85$ & $0-14$ & \\
\hline & AL1e $(60 \%)$ & $50-85$ & $5,5-14$ & \\
\hline & LL1b (20\%) & 30 & 70 & $\begin{array}{l}\text { Fundo arenoso com } \\
\text { alto teor de lama }\end{array}$ \\
\hline \multirow{3}{*}{$\begin{array}{c}2 \mathrm{~B} \\
\text { (c/ pene tração do } \\
\text { sinal c/ ocorrência } \\
\text { de refletores) }\end{array}$} & LL1c $(20 \%)$ & 20 & 80 & \\
\hline & LL1c $(85 \%)$ & $1-18$ & $80-99$ & $\begin{array}{l}\text { Fundo lamoso com } \\
\text { alto teor de areia fina } \\
\text { a muito fina }\end{array}$ \\
\hline & LL1b $(15 \%)$ & $27-36$ & $60-70$ & \\
\hline
\end{tabular}

Tabela 1: Relação dos tipos de ecocaráters e sedimentos.

Table 1: Types of echocharaters and sediments.

sedimento, e ocorrem onde o teor de matéria orgânica supostamente é alto. Infelizmente não foi feita nenhuma análise para verificar o teor de matéria orgânica nas amostras, no entanto, a literatura tem demonstrado o grande aporte de matéria orgânica na Baía de Guanabara.

\section{CONCLUSÕES}

A integração de dados geofísicos e sedimetológicos da área de estudo contribuiu para o entendimento dos processos sedimentares atuantes na região, podendo-se então delimitar áreas com características distintas dentro do sistema.

Da entrada do sistema até as proximidades da ponte Rio-Niterói, a baía sofre uma grande influência da morfologia, onde o estreitamento da entrada da barra bem como a existência de um banco de areia (Fig. 19) promovem um aumento da velocidade das correntes de maré. Este processo promove um selecionamento do sedimento impedindo a sedimentação lamosa e promovendo um retrabalhamento de areias médias de fundo. Nessa área observa-se o ecocaráter do tipo 1 e a existência de um campo de sand waves e ripple marks, indicadores de uma dinâmica atuante.

A progressiva diminuição da granulometria, desde areias finas a muito finas até lamas em direção ao interior do sistema ou em direção às áreas mais protegidas no interior das enseadas, é função da diminuição das correntes próximas ao fundo. Nessas áreas os ecocaráteres predominantes são do tipo $2 \mathrm{~A}$, $2 \mathrm{~B}$ e 3 .

Os ecocaráteres do tipo $2 \mathrm{~A}$ e 3 ocorrem em áreas com cobertura sedimentar lamosa (fundo da enseada de Jurujuba, Baptista Neto (1993) e próximo à ponte Rio-Niterói e adjacências da cidade de Niterói, onde a 
dinâmica das ondas e correntes de maré são pouco significativas na maior parte do tempo.

O ecocaráter do tipo 2B ocorre em fundo arenoso com alto teor de lama, próximo à ponte, e o tipo de reflexão de sonar em sua maior parte é de cinza escuro.

Como conclusão geral, vale salientar que, tanto o tipo de ecocaráter, como o padrão de reflexão do sonar de varredura lateral e os sedimentos de fundo têm uma boa correlação, mostrando serem importantes ferramentas para o estudo da dinâmica de sedimentos de uma área.

\section{AGRADECIMENTOS}

Ao Banco Nacional de Equipamentos Geofísicos, pelo empréstimo dos equipamentos. À DHN-RJ pela sessão dos registros de sonar de varredura e dados de sedimentos. Ao Dr. Cleverson Guizan Silva pelas críticas e apoio, aos revisores da RBGf pela discussão que muito contribuiu para o texto final. Ao LAGEMAR pela sessão dos dados de levantamentos realizados pelas turmas do curso de especialização em Geologia e Geofísica Marinha, e a CAPES, pelo financiamento da pesquisa, através de uma bolsa de mestrado.

\section{REFERÊNCIAS}

AMADOR, E.S. - 1992 - Sedimentos de fundo da Baía de Guanabara - Uma síntese. Anais do III Congresso ABEQUA - Belo Horizonte. 199224pp.

AYRES NETO, A \& AGUIAR, A. C. K. V. - 1993 Interpretação de reflexões de Side Scan Sonar: uma proposta de nomenclatura e padronização de métodos. Anais do Congresso Internacional de Geofísica RJ.1: 399-403

BAPTISTA NETO, J.A. - 1993 - Sedimentologia e geomorfologia da enseada de Jurujuba (Baía de Guanabara) - Niterói/RJ. Dissertação de Mestrado LAGEMAR - UFF.

BAPTISTA NETO, J.A., SILVA, M.A.M. \& FIGUEIREDO JR., A.G. - 1996 - Sísmica de alta freqüência e o padrão de distribuição de sedimentos na enseada de Jurujuba (Baía de Guanabara) - RJ/Brasil - Revista Brasileira de Geofísica 14(1): 51-57.

CALLIARI, L. J., ESTEVES, L. S., OLIVEIRA, C. P., TOZZI, H. A. M., SILVA, R. P. \& CARDOSO, J. N. - 1994 - Padrões sonográficos e sedimentológicos de um afloramento de beachrock na plataforma interna do Rio Grande do Sul (COMEMIR/OSNLR). Notas Técnicas, 7: 27-32.

COSTA, E.A. \& FIGUEIREDO Jr., A.G. - 1996 Ecocaráter da cobertura sedimentar da plataforma norte brasileira Anais do XXXIX Congresso Brasileiro de Geologia, Vol.3.

DAMUTH, J.E. - 1975 - Echo caracter of the western equatorial Atlantic floor and its relationship to the dispersal and distribution of terrigenous sediments. Marine Geology, 18: 17-45.

DAMUTH, J.E. \& HAYES, D.E. - 1977 - Echo caracter of east brazilian continental margin and its relationship to sedimentary processes. Marine Geology, 24:73-95.

DIAS, G.T.M. - 1996 - Classificação de sedimentos marinhos proposta de representação em cartas sedimentológicas. Anais do XXXIX Congresso Brasileiro de Geologia Vol.3.

Dias, G.T.M. \& Quaresma, V.S. - 1996 - Baia de Guanabara - Evolução Geomorfológica do Fundo Submarino. Anais do XXXIX Congresso Brasileiro de Geologia. Salvador, BA.

DRM - 1981 - Carta Geológica. Folha Baía de Guanabara, escala 1:50000.

FERRARI, A.L. - 1990 - A Geologia do "Rift" da Guanabara (RJ) na sua porção centro-ocidental e sua relação com o embasamento Pré-Cambriano. XXXVI Congresso Brasleiro de Geologia. Natal/ RN. 2858-2872pp.

FIGUEIREDO Jr. A.G., NITTROUER, C.A. \& COSTA, E.A. - 1993 - Gassy sediment in the Amazon submarine delta. Anais do III Congresso Internacional da Sociedade Brasileira de Geofísica.Rio de Janeiro - RJ/Brasil.1243-1247pp.

FLOOD, R.D. - 1980 - Deep sea sedimentary morphology: modelling and interpretation of echosouding profiles. Marine Geology, 38: 77-92.

HOLLISTER, C.D. \& HEEZEN, B.C. - 1972 Geologic effects of ocean bottom currents: western North Atlantic. In: A.L. Gordon (Eds.), Studies in Physical Oceanografy, 2, Gordon \& Breach, New York, N.Y., 37-66.

KJERFVE, B., RIBEIRO, C.H.A., DIAS, G.T.M., FILIPPO, A.M. \& QUARESMA, V.S. - 1997 Oceanographic characteristics of an impacted coastal bay: Baía de Guanabara, Rio de Janeiro, Brazil .Continental Shelf Research. 17,(13):16091643.

LARSONNEUR, C. - 1977 - La cartographie de's dépots meubles sur le plateau continental français: méthode mise du points et utilisée em Manche. 
Journal Redi Oceanog, 2:34-39.

MANLEY, P.L. \& FLOOD, R.D. - 1989 - Anomalous sound velocities in near-surface, organic-rich, gassy sediments in the Central Argentina basin. Deep-sea Research. 36(4):661-623.

PERILLO, G.M.E. - 1995 - Geomorphology and Sedimentology of Estuaries. 2nd ed. Elsevier. 471pp.
QUARESMA, V.S. - 1997 - Caracterização da dinâmica sedimentar da Baía de Guanabara, RJ. Dissertação de Mestrado - LAGEMAR -UFF. Niterói. 97pp.

TUCHOLKE, B.E., BRYAN, G.M. \& EWING, J.L. - 1977 - Gas hydrate horizons detected in seismicprofiler data from Western North Atlantic. AAPG Bulletin, 61: 698-707.

\section{CHARACTERIZATION OF SIDE-SCAN SONAR AND HIGH RESOLUTION SEISMIC (3.5 - $7.0 \mathrm{kHZ)}$ REFLECTION PATTERNS ALONG THE SOUTHERN MARGIN OF THE GUANABARA BAY - RJ}

The Guanabara Bay is a coastal plain estuary showing, in terms of salinity and water circulation, well-mixed estuary conditions in the bay mouth and partially mixed estuary nearby the Rio-Niterói Bridge. The goal of this article is to investigate the sediment processes from the Guanabara Bay (Rio de Janeiro - Brazil), focusing in the bottom morphology and sediment distribution. This goal has the purpose of integrating geomorphological, geological and geophysical characteristics in order to provide basic knowledge for the development of other projects in the Guanabara Bay. In terms of bottom morphology, the Guanabara Bay is characterised by a central channel extending up to the east of Governador Island, with North-South direction and depths ranging from 30 to $40 \mathrm{~m}$. Channel's morphology varies along it. Nearby the Rio-Niterói Bridge, it is deep and narrow, but northward, it becomes progressively shallower. A sand bank exists seaward of the bay mouth and waves are observed along the eastern margin of the central channel. The sand wave system is a function of SSW waves, associated with cold fronts, which set in movement bottom sediments available to be reworked by flood tidal bottom currents. The geophysical data analysis revealed six distinct reflection patterns from the side-scan sonar and four ecocharacters from $3.5 \mathrm{kHz}$. The side-scan reflection patterns are: homogeneous light grey associated with sands; homogeneous dark grey related with muddy bottom and medium sands; sand waves and megaripples, isolate targets type 1 and isolate targets type 2 occurring at 1200 $\mathrm{m}$ south and north of the bridge. The ecocharacters were classified as Type 1, showing no penetration and associated with a sandy bottom; Type 2a showing reflectors and the acoustic basement, is related to a muddy bottom with high weight (\%) of fine sand; Type $2 \mathrm{~b}$ presents sign penetration and is related to a sandy bottom with high weight (\%) of mud and type 3 with sign penetration and multiples, is associated with a muddy bottom with high weight (\%) of fine sand. The data analysis revealed that sediment distribution reflects tidal currents energy near the bottom, which is directly influenced by the bottom morphology and the Guanabara Bay contour. The strongest tidal currents are observed at the major choking points of the bay such, as the system mouth. The lowest energy tidal currents occur where the bay's contour becomes wider such, as within the Sounds or at its upper limits. Therefore, medium sized sands are distributed from the system mouth up to the south of Rio-Niterói bridge, where a progressive gradation to finer sediments are observed up to the occurrence of muds near the bridge, extending to the upper estuary boundaries.

\section{NOTE ABOUT THE AUTHORS}

\section{Valéria da Silva Quaresma}

Received her BSc in Geography and MSc in Marine Geology and Geophysics from the Universidade Federal Fluminense. She is currently at the
University of Southampton, the School of Oceanography working on her doctoral thesis about sediment dynamics. 


\section{Gilberto Tavares de Macedo Dias}

Received his BSc in Geology from the Universidade Federal do Rio de Janeiro. He concluded his doctoral thesis in 1976 at the Université de Bordeaux I. France and has been working on Marine Geology and Geophysics at the Universidade Federal Fluminense

\section{José Antônio Baptista Neto}

Received his BSc in Geography, Dipl. and MSc in Marine Geology and Geophysics from the Universidade Federal Fluminense. He concluded his Ph.D. in 1996 at The Queen's University of Belfast/UK and he has been working on coastal dynamics and evolution at the Universidade Federal Fluminense and Universidade do Estado do Rio de

\title{
UNIVERSIDADE FEDERAL FLUMINENSE DEPARTAMENTO DE GEOLOGIA
}

\section{CURSO DE PÓS GRADUAÇÃO EM GEOLOGIA E GEOFÍSICA MARINHA}

\author{
Nível: Mestrado
}

Clientela: Graduados em Geociências, Oceanografia e áreas afins.

Área de Concentração: Geologia e Geofísica Marinha

Objetivos do Curso: Capacitar profissionais para a execução de estudos, levantamentos, pesquisa pura e aplicada e para o exercício do magistério, na área de Geologia e Geofísica de Regiões Costeiras e Oceânicas.

\section{Corpo Docente:}

Alberto G. de Figueiredo Júnior, Doutor, Univ. de Miami, EUA

Cleverson Guizan Silva, Doutor, Univ. de Duke, EUA

Gilberto T. de Macedo Dias, Doutor, Univ. de Bordeaux 1, França

Isa Brehme, Doutor, Univ. de Bremen, Alemanha

Jorge Jesus Cunha Palma, Doutor, USP

Luiz Antonio P. Gamboa, Doutor, Univ. de Columbia, EUA

Marco Polo P. Boa Hora, Doutor, Univ. de Saint Louis, EUA

Marcus Aguiar Gorini, Doutor, Univ. de Columbia, EUA

Sidney Luiz de Matos Mello, Doutor,University of Leeds, Reino Unido

Susanna Eleonora Sichel, Doutor, Univ. de Rhode Island, EUA

\section{Endereço:}

\author{
Universidade Federal Fluminense \\ Instituto de Geociências \\ Curso de Pós-Graduação em Geologia e Geofísica Marinha \\ Av. Litorânea $\mathrm{s} / \mathrm{n}^{\circ}$ - Campus da Praia Vermelha \\ Boa Viagem - Niterói - RJ 24210-340 \\ Telefax: (021)719-4241 r.201 \\ Telex: 2132076UVFLBR \\ alberto@igeo.uff.br
}

